\title{
Assessing colorectal cancer mismatch repair status in the modern era: a survey of current practices and re-evaluation of the role of microsatellite instability testing
}

\author{
Erika Hissong ${ }^{1} \cdot$ Elizabeth P. Crowe ${ }^{1} \cdot$ Rhonda K. Yantiss $^{1} \cdot$ Yao-Tseng Chen ${ }^{1}$
}

Received: 9 April 2018 / Revised: 24 May 2018 / Accepted: 24 May 2018 / Published online: 28 June 2018

(c) United States \& Canadian Academy of Pathology 2018

\begin{abstract}
Results of DNA mismatch repair testing are used to detect Lynch syndrome and have prognostic and therapeutic implications among patients with sporadic colorectal carcinomas. Immunohistochemistry for mismatch repair proteins (MLH1, PMS2, MSH2, MSH6) and PCR for microsatellite instability are two established methods for assessing mismatch repair function. Older literature suggested a discordance rate of approximately $5 \%$ between these assays, leading some institutions to perform dual testing on all cases. Although universal mismatch repair testing is now recommended by multiple professional organizations, none provide guidelines regarding preferred assays. We surveyed 96 academic and nonacademic institutions to assess Lynch syndrome screening practices and evaluated discordance rates between immunohistochemistry and PCR among 809 colorectal cancers tested in our own institution. Our survey demonstrated no significant differences between academic and nonacademic practices with respect to testing strategies. Eighty six percent performed universal screening, and usually (76\%) employed immunohistochemistry on initial biopsy samples. Only $20 \%$ employed PCR; these were mostly academic practices that used both immunohistochemistry and PCR $(p<0.01$ compared with the nonacademic groups). Loss of MLH1/PMS2 staining was often (90\%) followed by either BRAF mutational analysis or MLH1 methylation assays. Only $24 \%$ adhered to WHO recommendations to assign histologic grade based on mismatch repair status. We found only 3 cases $(0.4 \%)$ with discordant immunohistochemistry and PCR results in our own practice: 1 reflected decreased MSH-6 staining in a neoadjuvantly treated microsatellite stable tumor, 1 MLH1-deficient tumor showed diminished MLH1/ PMS2 in the tumor compared with internal control, and 1 case reflected an error in the molecular laboratory. Overall, our results showed extremely low discordance between methods assessing mismatch repair status and would suggest immunohistochemistry as the preferred single screening test. PCR can be reserved for cases that show equivocal immunostaining patterns.
\end{abstract}

\section{Introduction}

Approximately $15-18 \%$ of colorectal carcinomas show mismatch repair deficiency [1-4]. Most of these are sporadic tumors with epigenetic silencing of $M L H 1$ expression through promoter hypermethylation or, less commonly,

Electronic supplementary material The online version of this article (https://doi.org/10.1038/s41379-018-0094-7) contains supplementary material, which is available to authorized users.

Yao-Tseng Chen

ytchen@med.cornell.edu

1 Department of Pathology and Laboratory Medicine, Weill Medical College of Cornell University, New York, NY 10065, USA somatic biallelic inactivation of mismatch repair genes. Up to $3 \%$ of tumors develop in association with Lynch syndrome and result from germline mutations in mismatch repair genes, most commonly MLH1, MSH2, MSH6, or PMS2 [5]. Guidelines aimed at detecting Lynch syndrome, such as the Amsterdam and Bethesda criteria, emphasize patient age, personal and family cancer history, and histologic features characteristic of mismatch repair-deficient colorectal cancers [6-9]. Unfortunately, screening criteria do not capture all affected individuals. At least $25 \%$ of patients with Lynch syndrome do not meet criteria prompting mismatch repair testing, and approximately $50 \%$ of patients with germline mutations develop their first Lynch-related cancers after the age of 50 years $[10,11]$. These observations, combined with the increasingly apparent therapeutic and prognostic importance of mismatch repair status [12-16], 
have led many professional organizations to recommend universal screening of all newly diagnosed colorectal cancers for mismatch repair deficiency.

Several different techniques can be used to assess mismatch repair status. Immunohistochemistry for MLH1, MSH2, MSH6, and PMS2 represents a qualitative measure of protein expression. It is widely available, relatively inexpensive, readily interpretable in most instances, and easily applied to biopsy or resection material. Polymerase chain reaction (PCR) for microsatellite instability indirectly measures functionality of the mismatch repair protein apparatus. Tumors with defective mismatch repair mechanisms accumulate replicative errors in short, repetitive sequences (i.e., microsatellites) that result in allelic shifts compared with non-tumor DNA from the same patient $[17,18]$. Finally, next-generation sequencing assays can indirectly measure mismatch repair status; mismatch repair-deficient tumors usually have a hypermutated phenotype compared with mismatch repairproficient tumors. Reported discordance rates between PCR and immunohistochemistry range up to nearly $10 \%$ in some studies leading many institutions, including our own, to employ both methods when assessing colorectal cancers for possible Lynch syndrome [19].

Although multiple professional organizations have endorsed universal testing of colorectal cancers for mismatch repair deficiency, available data suggest that many laboratories do not adhere to these recommendations. Moreover, specific guidance regarding preferred methodology is lacking. The purposes of this study were two-fold. First, we sought to identify the current state of affairs regarding mismatch repair testing of colorectal carcinomas and determine whether there were any differences between various practice types. We provided pathologists in 185 community and academic settings with a questionnaire survey to gauge their practices with respect to Lynch syndrome screening and colorectal cancer reporting. Second, we suspect that reported discordance rates between immunohistochemistry and PCR reflect imperfections of dinucleotide-based PCR panels and unfamiliarity with nuances of immunohistochemistry interpretation that have been largely overcome in current practice. Thus we evaluated our own experience with 809 colorectal cancers subjected to dual testing with immunohistochemistry for mismatch repair proteins and PCR.

\section{Materials and methods}

\section{Survey regarding mismatch repair deficiency testing of colorectal carcinomas}

We designed an 11-item questionnaire to query clinical practices regarding mismatch repair deficiency testing of colorectal carcinomas (see Supplementary Information 1). We sent the survey to pathologists at 185 academic and nonacademic practices, including 163 in the United States and 22 in Canada, Europe, and Australasia. A lack of response from the contact pathologist was followed by repeated attempts at contact and follow-up with another member of the group, as necessary. In order to avoid overrepresentation of larger practices, responses from only one pathologist at each institution were recorded. Pathologists were queried regarding selection criteria for screening, test of choice (e.g., immunohistochemistry for mismatch repair proteins and/or PCR), utilization of follow-up testing for abnormal results, and compliance with World Health Organization (WHO) recommendations regarding relationships between mismatch repair status and histologic grade assignment. To explore whether testing algorithms were influenced by financial reimbursement policies for inpatient testing, pathologists were also asked whether they were familiar with their institutional policies regarding financial reimbursement for ancillary studies. Multiple-choice answers were provided for each question and space was available for comments. Responses from each participating institution were collected, and the data were analyzed as a whole, as well as by practice type. Comparisons among practice models were performed using Chi-squared test. A $p$ value of $<0.05$ was considered statistically significant.

\section{Comparison between immunohistochemistry and PCR for Lynch syndrome screening}

We retrospectively identified all colorectal carcinoma cases subjected to mismatch repair-deficiency testing at New York-Presbyterian Hospital/Weill Cornell Medicine from January 2009 to June 2017. We identified 922 resected colorectal adenocarcinomas, including 103 cases evaluated by immunohistochemistry alone, 10 cases assessed by PCR alone, and 809 tumors evaluated with both immunohistochemistry for MLH1, PMS2, MSH2, and MSH6 and PCR using five mononucleotide markers. Cases that showed discordance between immunohistochemical results and microsatellite status were subjected to additional testing. In these cases, both immunohistochemistry and PCR were repeated on the blocks that were originally tested, as well as on an additional block of the tumor.

\section{Immunohistochemistry for mismatch repair protein expression}

Immunohistochemical stains were performed to evaluate MLH1 (clone G168-728, BD Biosciences, San Jose, CA), PMS2 (clone A16-4, BD Biosciences, San Jose, CA), MSH2 (clone FE11, Calbiochem, Burlington, MA), and MSH6 (clone 44/MSH6, BD Biosciences, San Jose, CA) 
using standard techniques. Loss of staining was defined as complete loss of nuclear staining in all of the tumor nuclei with preserved staining of lymphocytes and/or nonneoplastic crypt epithelium. Decreased staining intensity of tumor cells was noted when the internal control showed strong nuclear staining.

\section{Microsatellite instability testing by PCR}

Formalin-fixed paraffin-embedded sections of the tumor that showed at least $25 \%$ neoplastic cellularity and normal tissue controls were macrodissected and subjected to DNA extraction using the QIAamp DSP DNA FFPE Tissue Kit (Qiagen USA, Germantown, MD). Microsatellite status was determined using the Promega Microsatellite Instability Analysis System (Promega, Madison, WI) consisting of five nearly monomorphic mononucleotide markers (BAT-25, BAT-26, NR-21, NR-24, and MONO-27) and two highly polymorphic pentanucleotide markers (Penta $\mathrm{C}$ and Penta D) as identity controls. The PCR products were analyzed by capillary electrophoresis performed on the ABI 3130xl PRISM Genetic Analyzer with the Genescan Analysis GeneMapper Software version 4.0 (Applied Biosystems, Foster City, CA). Tumors were deemed microsatellite stable when there was no detectable shift in the electrophoretic pattern of the tumor DNA compared with that of corresponding normal tissue. The appearance of additional peaks in the tumor was interpreted to represent microsatellite instability. Tumors with instability at two or more markers were classified as microsatellite instability, whereas those with instability at only one marker were considered to have microsatellite instability.

\section{Results}

\section{Survey regarding screening practices for Lynch syndrome}

We received 96 responses from pathologists practicing in the United States $(n=84)$, Canada $(n=4)$, Europe $(n=5)$, and other international programs $(n=3)$ representing an overall survey response rate of $52 \%$. Respondents described their practice setting as academic $(n=59,61 \%)$ or nonacademic ( $n=37,39 \%$ ); the latter included community-based practices $(n=29,30 \%)$, private reference laboratories $(n=5$, $5 \%)$, and non-reference private laboratories $(n=3,3 \%)$. The survey results, detailed in Supplemental Information 2, are enumerated in Tables 1 and 2 and summarized below.

Eighty three $(86 \%)$ respondents reported universal screening of all colorectal cancers, and $56(67 \%)$ of these performed testing on biopsy specimens obtained at the time of cancer diagnosis. The overall universal screening rate was slightly higher among academic practices compared with nonacademic groups (92 and 78\%, respectively, $p=0.06$ ), but the differences were not significant, and both types of group performed their analyses on biopsy specimens at similar rates (59 and $57 \%$, respectively). Seven percent of practitioners did not perform universal testing on cancer specimens but reflexively tested samples from patients $<70$ years of age; only $4 \%$ relied on clinician requests to prompt screening. Nine percent of respondents performed universal screening on all extra-colonic adenocarcinomas of the gastrointestinal tract, while $54 \%$ screened such cases only upon clinician request and 19\% evaluated these tumors based on Bethesda guidelines. Of note, $76 \%$ of respondents did not consider mismatch repair status as a component of histologic grade assignment, regardless of criteria set forth by the WHO. A handful of respondents added commentary to the surgical pathology reports of histologically high-grade but mismatch repair-deficient cases, noting that mismatch repair-deficient tumors may behave less aggressively than mismatch repair-proficient tumors despite the presence of high-grade morphologic features.

Seventy nine percent of respondents screened their cases with immunohistochemistry for mismatch repair proteins alone, $18 \%$ routinely performed immunohistochemistry and PCR concurrently, and only a few institutions (2\%) performed PCR alone. Most $(96 \%)$ used an immunohistochemical stain panel consisting of four markers (MLH2, MSH2, MSH6, and PMS2), but three institutions screened cases with only MSH6 and PMS2. Combination testing with both immunohistochemistry and PCR was more common in academic institutions than in nonacademic centers (27 versus $3 \%$, respectively, $p<0.01$ ). Respondents from multiple academic institutions used next-generation sequencing to assess mutational burden as a surrogate marker of mismatch repair deficiency or noted that such methods were currently under development. Respondents from institutions that utilized both immunohistochemistry and PCR reported variable (estimated) discordance rates between the two methods. Eighteen percent estimated the discordance to be $<1 \%, 43 \%$ estimated discordance to be $1-5 \%$, and $36 \%$ estimated the discordance between immunohistochemical and molecular methods to be 5-10\%. Interestingly, $56 \%$ of respondents from academic institutions and $78 \%$ of those from nonacademic centers were not familiar with reimbursement policies regarding mismatch repair-deficiency testing in their institutions.

Many respondents reported follow-up molecular testing for tumors that showed combined loss of MLH1 and PMS2 immunostaining. Most (60\%) performed BRAF mutation analysis, $13 \%$ analyzed their cases for $M L H 1$ promoter hypermethylation, and $13 \%$ performed both of these tests simultaneously. Four percent performed subsequent testing only at the request of the clinician, and $6 \%$ did not offer ancillary testing beyond immunohistochemistry. Of respondents who performed $B R A F$ mutational testing when 
过导艺艺艺这 そ

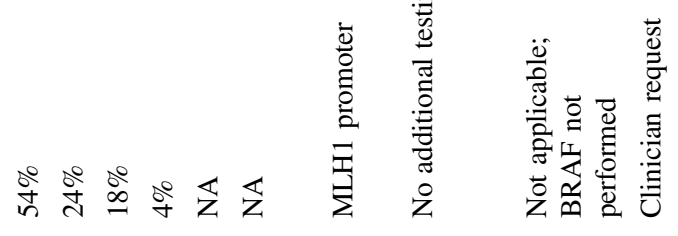

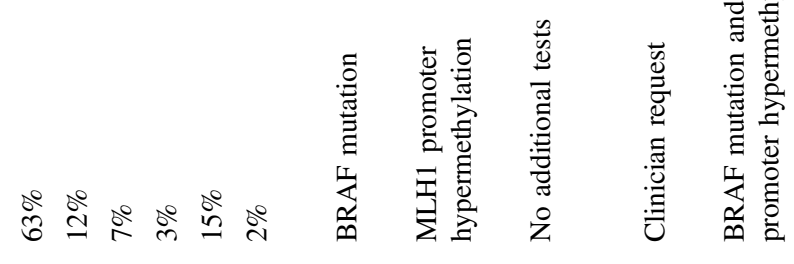

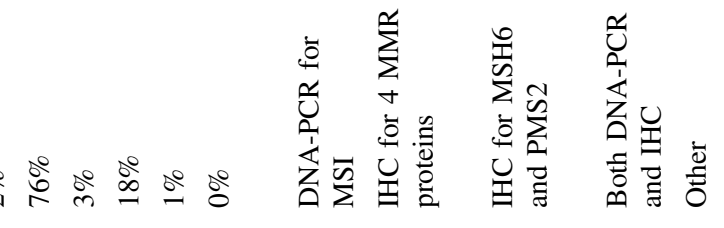

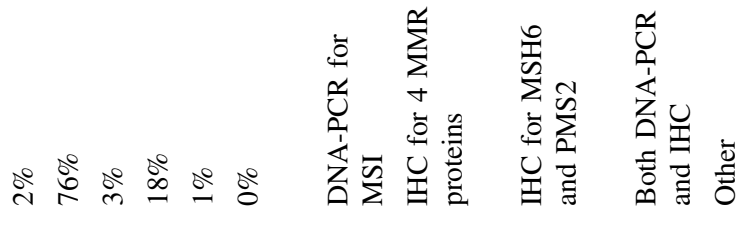

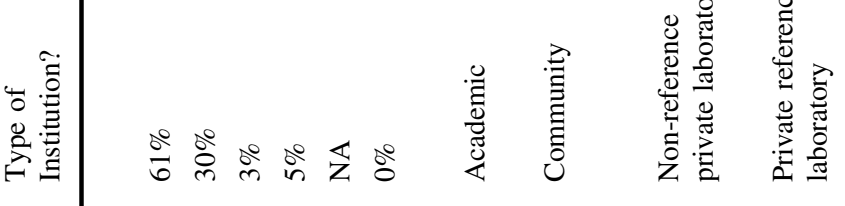

音

可.

$\Xi$

$\cong 0$

政

㟢通

恶迹

ส

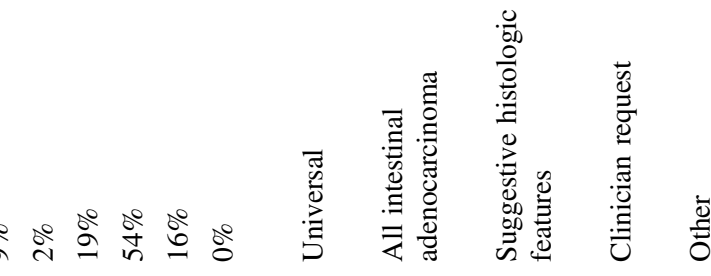

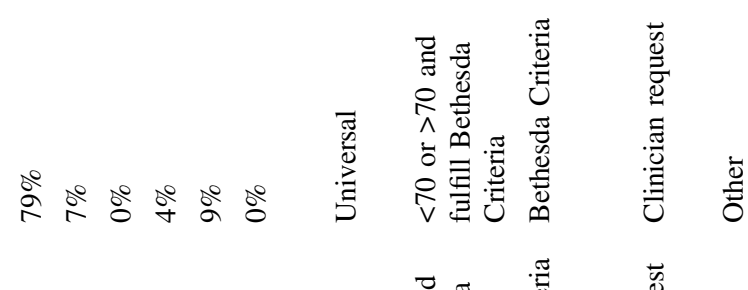

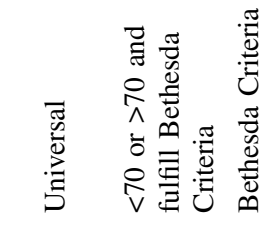

in

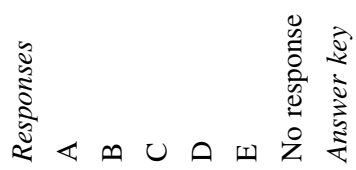

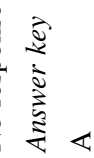

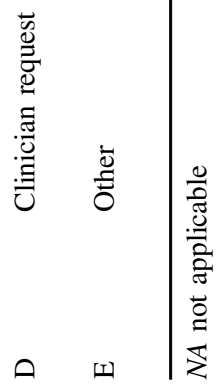


tumors showed immunohistochemical loss of MLH1 and PMS2, 87\% further evaluated their cases for $M L H 1$ promoter hypermethylation when wild-type $B R A F$ was detected. These practices were similar between academic and nonacademic institutions.

Table 2 Survey responses for MSI testing in academic and nonacademic institutions

\begin{tabular}{llll}
\hline & $\begin{array}{l}\text { Overall, } \\
N=96\end{array}$ & $\begin{array}{l}\text { Academic, } \\
N=59\end{array}$ & $\begin{array}{l}\text { Nonacademic, } \\
N=37\end{array}$ \\
\hline Universal screening & & & \\
Resection & $76(79 \%)$ & $49(83 \%)$ & $27(73 \%)$ \\
Biopsy & $56(58 \%)$ & $35(59 \%)$ & $21(57 \%)$ \\
Extra-colonic GI cancer & $10(10 \%)$ & $9(15 \%)$ & $1(3 \%)$ \\
Initial screening methodology & & & \\
IHC on MMR proteins only & $73(76 \%)$ & $39(66 \%)$ & $34(92 \%)$ \\
DNA-PCR only & $2(2 \%)$ & $2(3 \%)$ & $0(0 \%)$ \\
Both & $17(18 \%)$ & $16(27 \%)$ & $1(3 \%)$ \\
Subsequent testing for MLH1/MSH2 loss & & \\
BRAF mutation & $60(64 \%)$ & $37(63 \%)$ & $23(66 \%)$ \\
MLH1 hypermethylation & $12(13 \%)$ & $7(12 \%)$ & $5(14 \%)$ \\
Both & $12(13 \%)$ & $9(15 \%)$ & $3(9 \%)$ \\
Incorporation into histology grade & & \\
Yes & $23(24 \%)$ & $14(24 \%)$ & $9(25 \%)$ \\
No & $72(76 \%)$ & $45(76 \%)$ & $27(75 \%)$ \\
\hline
\end{tabular}

$* p<0.01$. All other comparisons between academic and nonacademic institutions showed no statistically significant difference

\section{Comparison between immunohistochemistry and PCR}

We analyzed 809 consecutive colorectal carcinoma resection specimens evaluated with both mismatch repair protein immunohistochemistry and PCR and compared the results. Concordant results were observed in $806(>99 \%)$ cases. Of these, $648(80.4 \%)$ showed preserved immunoexpression of all four mismatch repair proteins and showed either microsatellite stability $(n=645)$ or microsatellite instability $(n=3)$. The remaining $158(19.6 \%)$ concordant cases showed combined loss of MLH1 and PMS2 $(n=130$, $82.3 \%)$, loss of MSH2 and MSH6 ( $n=17,10.8 \%)$, isolated loss of PMS2 $(n=10,6.3 \%)$, or isolated loss of MSH6 ( $n=1,0.7 \%$ ) staining; all of these tumors showed microsatellite instability.

Only 3 cases $(0.4 \%)$ showed discordant results between immunohistochemistry and PCR. The first was a microsatellite stable tumor initially interpreted to be MSH6 deficient by immunohistochemistry, the second was a microsatellite stable tumor interpreted to show loss of MLH1/ PMS2 staining, and the third was a tumor with microsatellite instability interpreted to show preserved staining for all mismatch repair proteins. Two tumor blocks from each of these cases were subjected to additional immunohistochemistry for mismatch repair proteins and PCR (Table 3). Discordance in the first case was attributed to misinterpretation of the MSH6 immunohistochemical stain. The tumor was a neoadjuvantly treated rectal carcinoma with weak and patchy MSH6 staining (Fig. 1). Discordance in the second case was attributed to an

Table 3 Summary of discordant cases between MSI by DNA-PCR and IHC on MMR proteins

\begin{tabular}{lllll}
\hline & Original MSI-PCR & Repeat MSI-PCR & Original IHC interpretation & Repeat IHC interpretation \\
\hline Case \#1 & MSS & MSS & Loss of MSH6 & All 4 markers preserved \\
Case \#2 & MSS & MSI-H & Loss of MLH1 and PMS2 & Loss of MLH1 and PMS2 \\
Case \#3 & MSI-H & MSI-H & All 4 markers preserved & Staining for all 4 markers but MLH1 and PMS2 reduced \\
\hline
\end{tabular}
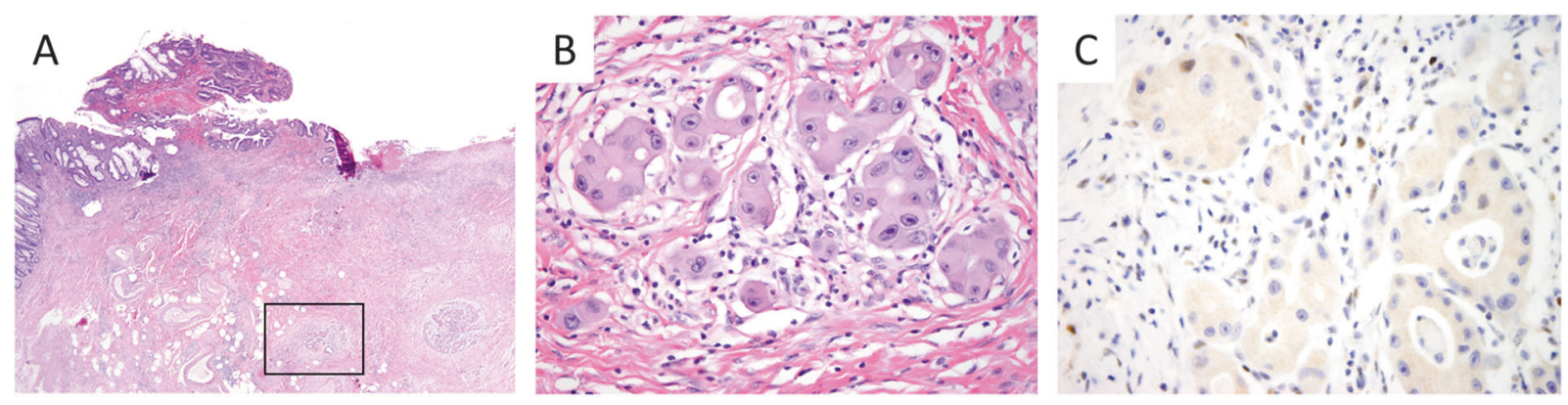

Fig. 1 This neoadjuvantly treated rectal adenocarcinoma consists of scattered clusters of tumor cells associated with extensive fibrosis (a, b). The MSH6 immunostain, originally interpreted as loss of staining compared with the internal control, shows nuclear staining in rare tumor cells (arrow) and this tumor is mismatch repair-proficient; decreased MSH6 staining is commonly seen in mismatch repair-proficient tumors following neoadjuvant therapy (c) 
Fig. 2 A mismatch repairdeficient adenocarcinoma of the sigmoid colon was originally interpreted to show retained mismatch repair protein staining. Non-neoplastic colonic mucosa and lymphoid aggregate (a) show diffuse strong nuclear staining for MLH1 (c) and PMS2 (e). In comparison, tumor cells (b) show positive but reduced and heterogeneous staining of both MLH1 (d) and PMS2 (f)
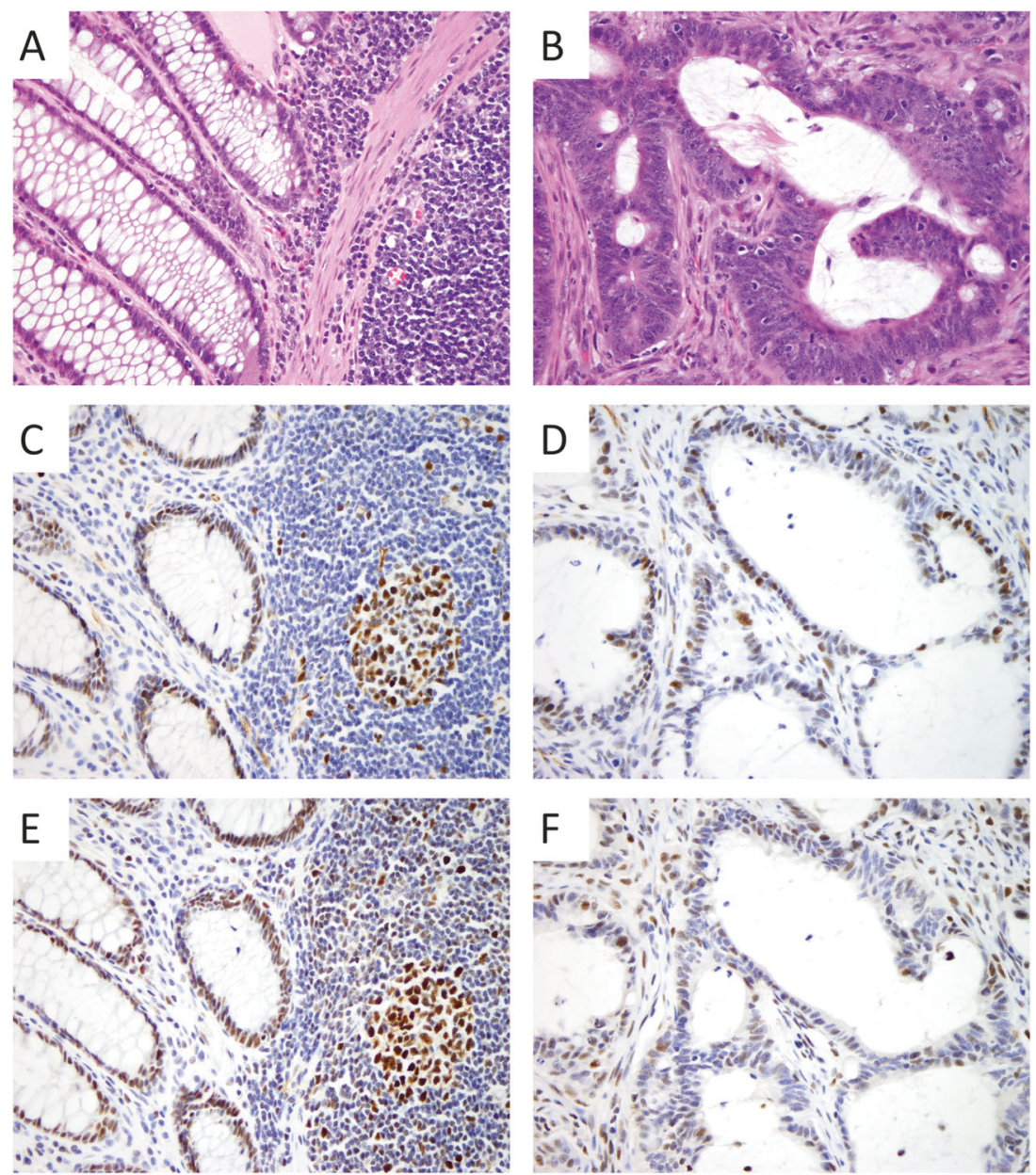

error in the molecular pathology laboratory. Repeat testing showed the tumor to be mismatch repair-deficient with concomitant loss of MLH1/PMS2 staining. The third discordant case was also attributed to an error in immunostain interpretation. Repeat testing by PCR demonstrated microsatellite instability, and the staining reactions for both MLH1 and PMS2 were significantly weaker and patchy in the tumor cells compared with adjacent non-neoplastic tissues on repeat immunohistochemistry; the MLH1 and PMS2 immunostains should have been interpreted as equivocal in this case (Fig. 2).

\section{Discussion}

The purposes of this study were to assess current practices with respect to screening colorectal carcinomas for Lynch syndrome and determine whether immunohistochemistry alone is sufficient to detect, or exclude, mismatch repair deficiency. We received feedback from 96 surveyed institutions and found $86 \%$ to employ universal testing of colorectal carcinomas for mismatch repair deficiency. Importantly, 66\% performed their analyses on biopsy specimens, thereby providing clinical colleagues an opportunity to incorporate test results into treatment planning. Although universal testing was more common among academic practices (92\%) compared with the private groups (78\%), the difference was not significant. Most institutions (79\%) relied on immunohistochemistry for mismatch repair proteins (MLH1, PMS2, MSH2, MSH6) alone and 2\% utilized PCR as the initial testing method; $18 \%$ used immunohistochemistry and PCR simultaneously. Tumors with combined loss of MLH1/PMS2 staining were subjected to BRAF mutational analysis and/or assessment for MLH1 promoter methylation by $90 \%$ of the practice groups. Financial considerations had no apparent bearing on test method selection, as most respondents were not familiar with institutional reimbursement policies for ancillary testing. Data from our own institution are also encouraging. We found a discordance rate of only $0.4 \%$ between mismatch repair protein immunohistochemistry and PCR among $>800$ tested cases. Furthermore, two of the three discordant cases were likely secondary to interpretative errors on re-examination; thus the true biologic discordant rate is as low as $1 / 809(0.1 \%)$ in our cohort. Our results paint a much more optimistic picture of current Lynch syndrome screening practices than can be inferred from data in the literature. Discordance rates 
between mismatch repair protein immunohistochemistry and PCR are sufficiently low to support immunohistochemistry as a single screening test, reserving other methods for cases that show equivocal immunostaining patterns.

Mismatch repair-deficiency testing was initially developed to screen cancer patients, mostly those with colorectal carcinoma, for Lynch syndrome, an autosomal-dominant heritable cancer syndrome resulting from germline alterations in mismatch repair genes. Screening criteria that rely on clinical and/or pathologic features to prompt testing fail to detect at least $10 \%$ of patients, leading several authors to advocate universal testing of colorectal cancer patients for Lynch syndrome [20-25]. This push towards universal testing gained momentum as the prognostic and predictive value of mismatch repair status among colorectal carcinomas and other solid tumors became evident. Mismatch repairdeficient tumors have a better prognosis compared with stage-matched mismatch repair-proficient tumors. In fact, the WHO defines histologic grade based on mismatch repair status; mismatch repair-deficient tumors are classified as low grade regardless of their morphologic features [14-16, 26]. Mismatch repair-deficient colorectal carcinomas also show a reduced response to fluorouracil-based chemotherapy compared with mismatch repair-proficient tumors and are more likely to respond to checkpoint-blockade immunoregulatory agents targeting programmed death 1 (PD1)-PD ligand 1 axis [12, 13, 27-29]. For all of these reasons, the U.S. MultiSociety Task Force on Colorectal Cancer, the National Comprehensive Cancer Network, National Society of Genetic Counselors, the Evaluation of Genomic Applications in Practice and Prevention Working Group, and many others advocate universal testing of all colorectal cancers for mismatch repair deficiency, although none make specific recommendations regarding test methodology or sample type [21, 24, 30-32].

Despite these recommendations, available data indicate that universal screening of colorectal cancers for Lynch syndrome is not widespread. Beamer et al. surveyed 80 institutions in the United States in 2012 and found that only $42 \%$ of respondents performed some type of assessment for mismatch repair deficiency without a clinician-driven request; universal testing was employed by only $38 \%$ of respondents [33]. The authors found that universal testing was usually performed by pathologists in an academic setting who performed immunohistochemistry alone (48\%), a combination of immunohistochemistry and PCR (38\%), or PCR alone (14\%). Six years after publication of those data, our findings suggest encouraging trends among both academic and private practices. We found that $86 \%$ of institutions performed universal screening of colorectal cancers, and the gap between academic and community practices no longer exists. Our data also demonstrate an overall decrease in the use of PCR in recent years, especially as a single screening tool; we found that only $20 \%$ of practices utilized PCR compared with $52 \%$ in the prior survey [33].

Immunohistochemistry for mismatch repair proteins has several advantages over PCR. It is more widely available and less expensive than PCR, interpretation of stain results is generally straightforward, and the pattern of staining can be used as a surrogate marker of the underlying genetic defect, thereby guiding subsequent molecular studies. However, published discordance rates between these assays range from 1 to $9 \%$ [19, 34-40]. In fact, only $18 \%$ of respondents to our survey estimated the discordance rate between immunohistochemistry and PCR in their institutions to be $<1 \%$; most $(43 \%)$ estimated it to be $1-5 \%$, and $36 \%$ estimated a discordance rate of $5-10 \%$ between these testing methods.

Misclassification of tumors by PCR is more likely when microsatellite markers include both dinucleotide and mononucleotide repeats because alterations in the former are not as specific for mismatch repair deficiency as expansion or contraction of monomorphic mononucleotide repeats. Murphy et al. evaluated 34 colorectal cancers and found that 5 mismatch repair-proficient tumors initially classified as microsatellite instability-low on PCR by the Bethesda panel were re-classified as microsatellite stable when a commercially available panel of five quasi-monomorphic mononucleotide markers was used [18]. Carcinomas with mismatch repair deficiency resulting from MSH6 mutations may not be classified as microsatellite unstable when dinucleotide markers are used because MSH6 is not involved in the repair of mismatches only two nucleotides in length; MSH6-deficient tumors are often erroneously classified as microsatellite stable with the Bethesda panel [41].

Errors may also occur when screening is limited to immunohistochemical panels that do not include four antibodies. Debniak et al. reported $9 \%$ of mismatch repair-deficient tumors to show preserved immunostaining for mismatch repair proteins, but they only used antibodies to MLH1 and MSH2 to evaluate their cases [19]. Some authors have proposed that a twoantibody panel comprised of PMS2 and MSH6 is a sensitive, specific, and cost-effective alternative to the four-antibody panel as an initial screen for mismatch repair deficiency [42, 43]. Although some authors have reported that a panel consisting of only PMS2 and MSH6 detects mismatch repair deficiency with $100 \%$ sensitivity and specificity, those authors limited their analyses to cancers that showed histologic features suspicious for mismatch repair deficiency, occurred among patients aged $<40$ years, and those with increased risk for Lynch syndrome based on revised Bethesda criteria $[42,43]$. When applied to a general population, use of this two-marker panel failed to detect $4 \%$ of colorectal carcinomas with underlying mismatch repair deficiency in one study, possibly reflecting abnormalities in MLH1 or MSH2 that did not result in degradation of PMS2 or MSH6, respectively [44]. 
Fig. 3 Proposed algorithm for the evaluation of mismatch repair status in colorectal carcinoma

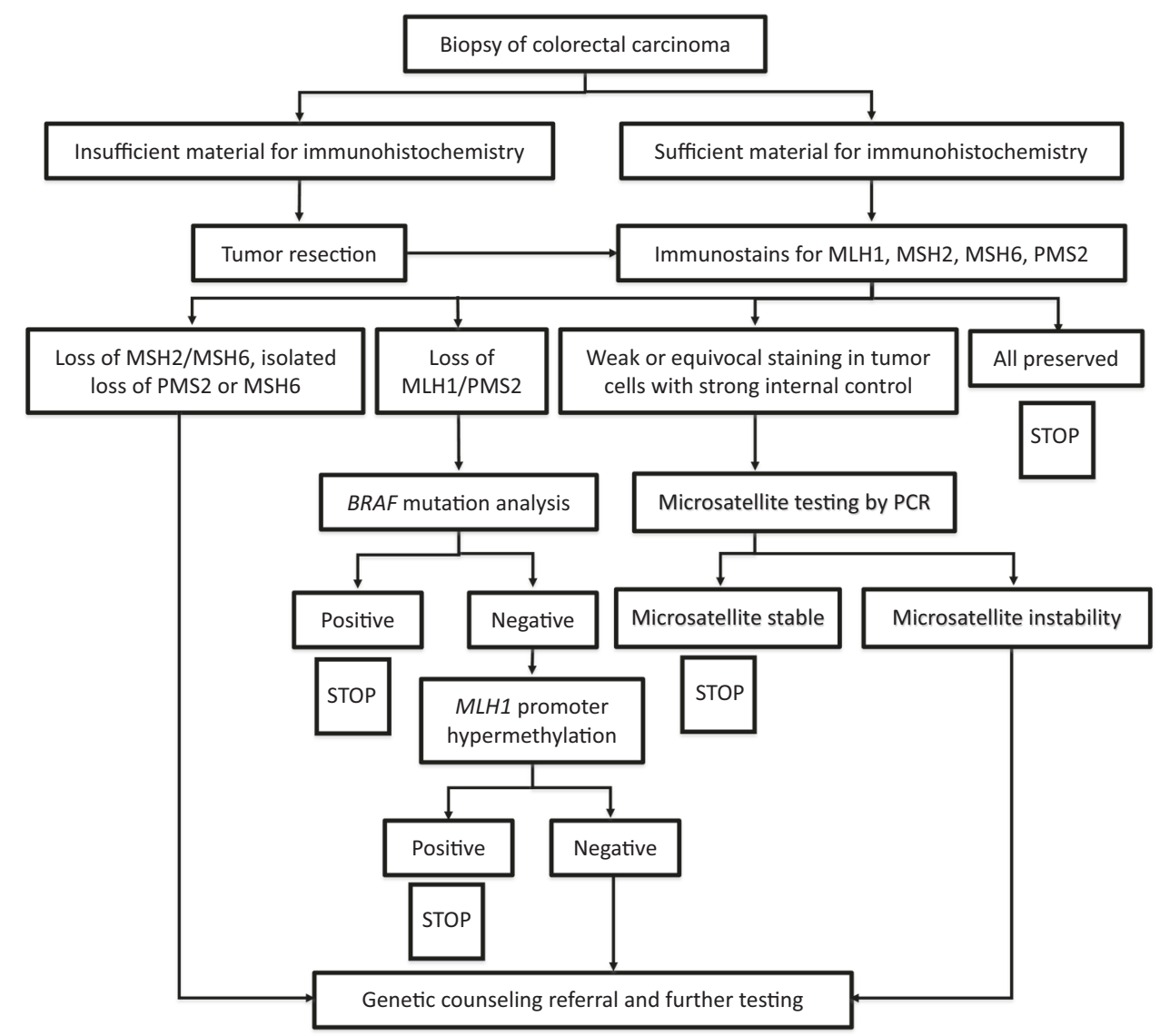

Lack of familiarity with nuances of mismatch repair protein immunohistochemistry can also lead to interpretive errors. Bao et al. found that patchy, often extensive loss of MSH6 was present in $20 \%$ of posttreatment rectal cancer resection specimens compared with pretreatment biopsy material [45]. Similarly, Vilkin et al. found PMS2 staining to be decreased in up to $30 \%$ of rectal carcinomas following treatment [46]. Decreased intensity of tumor cell staining may also reflect genetic alterations that produce non-functional mismatch repair proteins with partially retained immunoreactivity or defects in other component(s) of the DNA mismatch repair complex, such as MSH3 [19, 47-49]. Some cases with such genetic alterations will show weak/patchy staining compared with the internal positive control, as we observed in case \#3, allowing them to be identified as abnormal and followed with PCR or other methods [50, 51]. However, other cases may fully retain the expression of MLH1, MSH2, MSH6, and PMS2 by immunohistochemistry, resulting in true biologic discordance between immunohistochemistry and microsatellite PCR tests, with immunohistochemistry results being incorrect [38, 52-54]. We believe that the frequency of this false immunohistochemical result is extremely low, as we did not observe it in our $800+$ cases' cohort.

Next-generation sequencing is an emerging technique that simplifies testing algorithms and preserves tissue samples. In addition to providing information regarding the mutational status of $B R A F, K R A S$, and other cancerassociated genes, it detects the hypermutator phenotype due to either mismatch repair deficiency or alterations in DNA polymerase epsilon catalytic subunit (i.e., POLE mutations). Recent studies have developed criteria for determining mismatch repair status based on microsatellite markers sequenced through next-generation sequencing or assessing total mutational burden [55-59]. Although several respondents indicated that they utilize next-generation sequencing to determine mismatch repair status, this type of assay is still in development and its utility will likely be largely restricted to academic centers with sufficient resources to undergo such complex analyses on numerous samples routinely. In addition, performing next-generation sequencing on all stage 1 colorectal cancers for which Lynch screening is the sole purpose of mismatch repair status assessment may not be justified.

Mismatch repair-deficient colorectal carcinomas pursue a less aggressive clinical course than stage-matched mismatch repair-proficient tumors, and thus the WHO considers all mismatch repair-deficient colorectal carcinomas to be low grade, regardless of histologic features [18, 26, 60]. Our results indicate that most $(76 \%)$ pathologists do not follow these recommendations. The most likely explanation to this 
observation would be that many pathologists object to incorporating ancillary test results into histologic parameters and would prefer to consider mismatch repair status an independent prognostic factor. Emerging data suggest that histologic grade is prognostically important, even among mismatch repair-deficient colorectal tumors. Johncilla et al. evaluated 119 mismatch repair-deficient colonic carcinomas and found that histologically high-grade tumors were more often of advanced stage and associated with decreased survival [61]. The discrepancy between published recommendations and clinical practice should be addressed.

The results of this study support utilization of a fourmarker immunohistochemical panel (MLH1, PMS2, MSH2, MSH6) as a screening tool for mismatch repair deficiency among colorectal carcinomas, preferably biopsy samples, reserving PCR for cases that show equivocal staining patterns. Figure 3 shows the flowchart that we would propose for the assessment of mismatch repair status in cancer. As noted by others, loss of MLH1/PMS2 staining in a tumor should prompt BRAF mutation testing followed by $M L H 1$ promoter hypermethylation analysis for $B R A F$ wild-type cases. The rationale for testing $B R A F$ first is that $B R A F$ mutations can be detected in up to $70 \%$ of MLH1/PMS2deficient tumors and are virtually absent in Lynch syndromeassociated tumors, making $B R A F$ mutation testing a highly sensitive tool for excluding germline alterations when mutations are identified [62-65]. In addition, BRAF mutation testing is more readily available in most laboratories than methylation analysis and is more cost effective [66]. No further evaluation is necessary for cases with $B R A F$ mutations or $M L H 1$ promoter hypermethylation. Failure to detect these alterations in a tumor, loss of MLH1/PMS2 staining, or any other combination (e.g., loss of MSH2 and MSH6, loss of MSH6, and loss of PMS2) should prompt referral genetic counseling [67]. This streamlined workflow is efficient, cost effective, and timely enough to allow for incorporation of mismatch repair status into the surgical pathology report issued at the time of initial cancer diagnosis.

Acknowledgements The authors would like to thank the Weill Cornell Department of Pathology-Translational Research Program for funding and support, as well as the respondents for their participation in the survey and their thoughtful comments.

\section{Compliance with ethical standards}

Conflict of interest The authors declare that they have no conflict of interest.

\section{References}

1. Ionov Y, Peinado MA, Malkhosyan S, et al. Ubiquitous somatic mutations in simple repeated sequences reveal a new mechanism for colonic carcinogenesis. Nature. 1993;363:558-61.
2. Boland CR, Goel A. Microsatellite instability in colorectal cancer. Gastroenterology. 2010;138:2073.e3-87.e3.

3. Thibodeau SN, Bren G, Schaid D. Microsatellite instability in cancer of the proximal colon. Science. 1993;260:816-9.

4. Vilar E, Gruber SB. Microsatellite instability in colorectal cancerthe stable evidence. Nat Rev Clin Oncol. 2010;7:153-62.

5. Plazzer JP, Sijmons RH, Woods MO, et al. The InSiGHT database: utilizing 100 years of insights into Lynch syndrome. Fam Cancer. 2013;12:175-80.

6. Jenkins MA, Hayashi S, O'Shea AM, et al. Pathology features in Bethesda guidelines predict colorectal cancer microsatellite instability: a population-based study. Gastroenterology. 2007;133:48-56.

7. Wright CL, Stewart ID. Histopathology and mismatch repair status of 458 consecutive colorectal carcinomas. Am J Surg Pathol. 2003;27:1393-406.

8. Jass JR. Classification of colorectal cancer based on correlation of clinical, morphological and molecular features. Histopathology. 2007;50:113-30.

9. Gologan A, Krasinskas A, Hunt J, et al. Performance of the revised Bethesda guidelines for identification of colorectal carcinomas with a high level of microsatellite instability. Arch Pathol Lab Med. 2005;129:1390-7.

10. Hampel H. Point: justification for Lynch syndrome screening among all patients with newly diagnosed colorectal cancer. J Natl Compr Canc Netw. 2010;8:597-601.

11. de la Chapelle A, Palomaki G, Hampel H. Identifying Lynch syndrome. Int J Cancer. 2009;125:1492-3.

12. Benatti P, Gafa R, Barana D, et al. Microsatellite instability and colorectal cancer prognosis. Clin Cancer Res. 2005;11:8332-40.

13. Ribic CM, Sargent DJ, Moore MJ, et al. Tumor microsatelliteinstability status as a predictor of benefit from fluorouracil-based adjuvant chemotherapy for colon cancer. N Engl J Med. 2003; 349:247-57.

14. Gavin PG, Paik S, Yothers G, et al. Colon cancer mutation: prognosis/prediction--response. Clin Cancer Res. 2013;19:1301.

15. Popat S, Hubner R, Houlston RS. Systematic review of microsatellite instability and colorectal cancer prognosis. J Clin Oncol. 2005;23:609-18.

16. Guastadisegni C, Colafranceschi M, Ottini L, et al. Microsatellite instability as a marker of prognosis and response to therapy: a meta-analysis of colorectal cancer survival data. Eur J Cancer. 2010;46:2788-98.

17. Boland CR, Thibodeau SN, Hamilton SR, et al. A National Cancer Institute Workshop on Microsatellite Instability for cancer detection and familial predisposition: development of international criteria for the determination of microsatellite instability in colorectal cancer. Cancer Res. 1998;58:5248-57.

18. Murphy KM, Zhang S, Geiger T, et al. Comparison of the microsatellite instability analysis system and the Bethesda panel for the determination of microsatellite instability in colorectal cancers. J Mol Diagn. 2006;8:305-11.

19. Debniak T, Kurzawski G, Gorski B, et al. Value of pedigree/ clinical data, immunohistochemistry and microsatellite instability analyses in reducing the cost of determining hMLH1 and hMSH2 gene mutations in patients with colorectal cancer. Eur J Cancer. 2000;36:49-54.

20. Buza N, Ziai J, Hui P. Mismatch repair deficiency testing in clinical practice. Expert Rev Mol Diagn. 2016;16:591-604.

21. Cohen SA, Laurino M, Bowen DJ, et al. Initiation of universal tumor screening for Lynch syndrome in colorectal cancer patients as a model for the implementation of genetic information into clinical oncology practice. Cancer. 2016;122:393-401.

22. Lynch HT, de la Chapelle A. Hereditary colorectal cancer. N Engl J Med. 2003;348:919-32. 
23. Hampel H, Frankel WL, Martin E, et al. Screening for the Lynch syndrome (hereditary nonpolyposis colorectal cancer). N Engl $\mathbf{J}$ Med. 2005;352:1851-60.

24. Zhang X, Li J. Era of universal testing of microsatellite instability in colorectal cancer. World J Gastrointest Oncol. 2013;5: $12-19$.

25. Boland CR, Shike M. Report from the Jerusalem workshop on Lynch syndrome-hereditary nonpolyposis colorectal cancer. Gastroenterology. 2010;138:2191-7.

26. Bosman FT, Carneiro F, World Health Organization, International Agency for Research on Cancer (IARC). WHO classification of tumours of the digestive system. 4th ed. Lyon: IARC; 2010. p. 417.

27. Le DT, Uram JN, Wang H, et al. PD-1 blockade in tumors with mismatch-repair deficiency. N Engl J Med. 2015;372:2509-20.

28. Goldberg KB, Blumenthal GM, McKee AE, et al. The FDA Oncology Center of Excellence and precision medicine. Exp Biol Med (Maywood). 2017;243:308-312.

29. Overman MJ, McDermott R, Leach JL, et al. Nivolumab in patients with metastatic DNA mismatch repair-deficient or microsatellite instability-high colorectal cancer (CheckMate 142): an open-label, multicentre, phase 2 study. Lancet Oncol. 2017;18:1182-91.

30. Hampel H. NCCN increases the emphasis on genetic/familial high-risk assessment in colorectal cancer. J Natl Compr Canc Netw. 2014;12:829-31.

31. Weissman SM, Burt R, Church J, et al. Identification of individuals at risk for Lynch syndrome using targeted evaluations and genetic testing: National Society of Genetic Counselors and the Collaborative Group of the Americas on Inherited Colorectal Cancer joint practice guideline. J Genet Couns. 2012;21:484-93.

32. Funkhouser WK Jr., Lubin IM, Monzon FA, et al. Relevance, pathogenesis, and testing algorithm for mismatch repair-defective colorectal carcinomas: a report of the association for molecular pathology. J Mol Diagn. 2012;14:91-103.

33. Beamer LC, Grant ML, Espenschied CR, et al. Reflex immunohistochemistry and microsatellite instability testing of colorectal tumors for Lynch syndrome among US cancer programs and follow-up of abnormal results. J Clin Oncol. 2012;30:1058-63.

34. Bartley AN, Luthra R, Saraiya DS, et al. Identification of cancer patients with Lynch syndrome: clinically significant discordances and problems in tissue-based mismatch repair testing. Cancer Prev Res (Phila). 2012;5:320-7.

35. Shia J. Immunohistochemistry versus microsatellite instability testing for screening colorectal cancer patients at risk for hereditary nonpolyposis colorectal cancer syndrome. Part I. The utility of immunohistochemistry. J Mol Diagn. 2008;10:293-300.

36. Cawkwell L, Gray S, Murgatroyd H, et al. Choice of management strategy for colorectal cancer based on a diagnostic immunohistochemical test for defective mismatch repair. Gut. 1999;45: 409-15.

37. Dieumegard B, Grandjouan S, Sabourin JC, et al. Extensive molecular screening for hereditary non-polyposis colorectal cancer. Br J Cancer. 2000;82:871-80.

38. Lindor NM, Burgart LJ, Leontovich $\mathrm{O}$, et al. Immunohistochemistry versus microsatellite instability testing in phenotyping colorectal tumors. J Clin Oncol. 2002;20:1043-8.

39. Yuan L, Chi Y, Chen W, et al. Immunohistochemistry and microsatellite instability analysis in molecular subtyping of colorectal carcinoma based on mismatch repair competency. Int $\mathbf{J}$ Clin Exp Med. 2015;8:20988-21000.

40. Hampel H, Frankel WL, Martin E, et al. Feasibility of screening for Lynch syndrome among patients with colorectal cancer. J Clin Oncol. 2008;26:5783-8.

41. Wu Y, Berends MJ, Mensink RG, et al. Association of hereditary nonpolyposis colorectal cancer-related tumors displaying low microsatellite instability with MSH6 germline mutations. Am J Hum Genet. 1999;65:1291-8.

42. Shia J, Tang LH, Vakiani E, et al. Immunohistochemistry as firstline screening for detecting colorectal cancer patients at risk for hereditary nonpolyposis colorectal cancer syndrome: a 2-antibody panel may be as predictive as a 4-antibody panel. Am J Surg Pathol. 2009;33:1639-45.

43. Hall G, Clarkson A, Shi A, et al. Immunohistochemistry for PMS2 and MSH6 alone can replace a four antibody panel for mismatch repair deficiency screening in colorectal adenocarcinoma. Pathology. 2010;42:409-13.

44. Hicks SC, Ward RL, Hawkins NJ. Immunohistochemistry for PMS2 and MSH6 alone can replace a four antibody panel for mismatch repair deficiency screening in colorectal adenocarcinoma. Pathology. 2011;43:84-85. author reply 85-86.

45. Bao F, Panarelli NC, Rennert H, et al. Neoadjuvant therapy induces loss of MSH6 expression in colorectal carcinoma. Am J Surg Pathol. 2010;34:1798-804.

46. Vilkin A, Halpern M, Morgenstern S, et al. How reliable is immunohistochemical staining for DNA mismatch repair proteins performed after neoadjuvant chemoradiation? Hum Pathol. 2014;45:2029-36.

47. Ligtenberg MJ, Kuiper RP, Chan TL, et al. Heritable somatic methylation and inactivation of MSH2 in families with Lynch syndrome due to deletion of the 3' exons of TACSTD1. Nat Genet. 2009;41:112-7.

48. Orimo H, Nakajima E, Yamamoto M, et al. Association between single nucleotide polymorphisms in the hMSH3 gene and sporadic colon cancer with microsatellite instability. J Hum Genet. 2000;45:228-30.

49. Bellizzi AM, Frankel WL. Colorectal cancer due to deficiency in DNA mismatch repair function: a review. Adv Anat Pathol. 2009;16:405-17.

50. Klarskov L, Ladelund $\mathrm{S}$, Holck $\mathrm{S}$, et al. Interobserver variability in the evaluation of mismatch repair protein immunostaining. Hum Pathol. 2010;41:1387-96.

51. Fadhil W, Ilyas M. Immunostaining for mismatch repair (MMR) protein expression in colorectal cancer is better and easier to interpret when performed on diagnostic biopsies. Histopathology. 2012;60:653-5.

52. Sepulveda AR, Hamilton SR, Allegra CJ, et al. Molecular biomarkers for the evaluation of colorectal cancer. Am J Clin Pathol. 2017. doi: 10.1093/ajcp/aqw209. [Epub ahead of print] PMID:28165529

53. Watson N, Grieu F, Morris M, et al. Heterogeneous staining for mismatch repair proteins during population-based prescreening for hereditary nonpolyposis colorectal cancer. J Mol Diagn. 2007;9: 472-8.

54. Wahlberg SS, Schmeits J, Thomas G, et al. Evaluation of microsatellite instability and immunohistochemistry for the prediction of germ-line MSH2 and MLH1 mutations in hereditary nonpolyposis colon cancer families. Cancer Res. 2002;62: 3485-92.

55. Niu B, Ye K, Zhang Q, et al. MSIsensor: microsatellite instability detection using paired tumor-normal sequence data. Bioinformatics. 2014;30:1015-6.

56. Huang MN, McPherson JR, Cutcutache I, et al. MSIseq: software for assessing microsatellite instability from catalogs of somatic mutations. Sci Rep. 2015;5:13321.

57. Salipante SJ, Scroggins SM, Hampel HL, et al. Microsatellite instability detection by next generation sequencing. Clin Chem. 2014;60:1192-9.

58. Nowak JA, Yurgelun MB, Bruce JL, et al. Detection of mismatch repair deficiency and microsatellite instability in colorectal adenocarcinoma by targeted next-generation sequencing. J Mol Diagn. 2017;19:84-91. 
59. Stadler ZK, Battaglin F, Middha S, et al. Reliable detection of mismatch repair deficiency in colorectal cancers using mutational load in next-generation sequencing panels. J Clin Oncol. 2016;34:2141-7.

60. Rosty C, Williamson EJ, Clendenning M, et al. Should the grading of colorectal adenocarcinoma include microsatellite instability status? Hum Pathol. 2014;45:2077-84.

61. Johncilla M, Chen Z, Yantiss RK. Histologic grade is prognostically important among colorectal carcinomas regardless of microsatellite status. Mod Pathol. 2018;31:272.

62. Bouzourene H, Hutter P, Losi L, et al. Selection of patients with germline MLH1 mutated Lynch syndrome by determination of MLH1 methylation and BRAF mutation. Fam Cancer. 2010;9:167-72.

63. Domingo E, Laiho P, Ollikainen M, et al. BRAF screening as a low-cost effective strategy for simplifying HNPCC genetic testing. J Med Genet. 2004;41:664-8.
64. Parsons MT, Buchanan DD, Thompson B, et al. Correlation of tumour BRAF mutations and MLH1 methylation with germline mismatch repair (MMR) gene mutation status: a literature review assessing utility of tumour features for MMR variant classification. J Med Genet. 2012;49:151-7.

65. Pino MS, Chung DC. Application of molecular diagnostics for the detection of Lynch syndrome. Expert Rev Mol Diagn. 2010;10:651-65.

66. Mvundura M, Grosse SD, Hampel H, et al. The cost-effectiveness of genetic testing strategies for Lynch syndrome among newly diagnosed patients with colorectal cancer. Genet Med. 2010;12:93-104.

67. Pai RK, Pai RK. A practical approach to the evaluation of gastrointestinal tract carcinomas for Lynch syndrome. Am J Surg Pathol. 2016;40:e17-34. 